\title{
A BASIS FOR THE COMPARISON OF X-RAYS GENERATED BY VOLTAGES OF DIFFERENT WAVE FORM
}

\author{
By Lauriston S. Taylor, George Singer, and C. F. Stoneburner
}

\section{ABSTRACT}

Earlier studies of the relationship between the applied voltage and the emission of general X radiation for various voltage wave forms (B.S.Jour. Research, vol. 9 (RP505), p. 769, 1932) have been extended to include generators not previously available. The X-ray output per effective (rms) milliampere of tube current is found for full wave and constant potential to be nearly the same at any given value of the effective (rms) voltage. For half-wave generators it is necessary to make allowance for suppressed half cycle which is useless in the production of X-rays, but which enters in the measurement of current or voltage with an a.c. meter. Current and voltage measurements with half-wave rectifiers are corrected so as to apply only during the useful portion of the cycle by the application of a factor of $\sqrt{ } 1 / 2$ and $\sqrt{ } 2$ to the corresponding values measured over a whole cycle. The half-wave generator then yields results similar to the other generators. The X-ray quality, as expressed by a full absorption curve, is found to be the same for all wave forms having the same effective (rms) value, and regardless of peak values. It is thus possible to adequately express the quality of any radiation in terms of the effective voltage used to excite it. This in turn relates the quality directly to that produced by constant potential. Thus the difficulty in the use of a full absorption curve to express X-ray quality - namely, its inexpressibility as a single numerical magnitude - is overcome. All output measurements made in terms of peak voltage and average current are found to be without real significance in quality determinations.

\section{CONTENTS}

I. Introduction

II. Experimental procedure

III. Experimental results

2. Output per effective milliampere

3. Quality as related to voltage measurements............

4. Changes in voltage wave form

IV. Conclusion

\section{INTRODUCTION}

$\mathrm{X}$ radiation is necessarily and adequately specified physically by its intensity (quantity) and spectral distribution (quality, hardness).

The intensity is defined as the quantity of energy flowing through unit cross section of the beam in unit time. The spectral distribution is defined as the fractional part of the intensity comprised within a uniform narrow wave length (or frequency) interval throughout the X-ray spectrum.

Intensity is ideally measured in ergs per square centimeter per second. In practice, however, intensity is commonly expressed in roentgens per unit time, which are proportional to the electrical con- 
ductivity which the X-ray beam imparts to a cubic centimeter of air. This conductivity is, for any given wave length or any given spectral distribution, very closely proportional to the intensity. ${ }^{3}$ Since, however, the factor of proportionality varies with the wave length, the ionization chamber indications do not serve to give the relative intensities of different spectral distributions. The conductivity imparted to air by radiations having different spectral distribution is, probably, a better measure than intensity of their relative therapeutic value; hence, the conductivity of air, expressed in roentgens per unit time, has been internationally adopted as the physical unit of measure of an X-ray dose. But, even in dosage, it is important to realize that the same number of roentgens represents the same dose only when the spectral distribution of the radiations is the same.

The spectral distribution of intensity is represented by a curve, throughout the spectrum, plotted as a function of the wave length or the frequency. Its direct determination is cumbersome and subject to considerable error. In practice, it is inferred as quality (or "hardness") from the thickness of an absorber, say copper, required to reduce the number of roentgens in the radiation to one half. This thickness is called the half value layer. ${ }^{2}$ More complete information of the spectral distribution is inferred from the complete absorption curve, ${ }^{3}$ obtained with a series of thicknesses of the absorbing material. ${ }^{4}{ }_{6}$ In fact Silberstein, ${ }^{7}$ has shown that the complete spectral distribution curve neglecting characteristic lines is obtainable from an exact absorption curve.

It is well known that the half value layer falls short of satisfactorily expressing the quality of the radiation; and the complete absorption curve, though adequate, lacks the very desirable simplicity of being expressible as a numerical magnitude. The experimental results published in previous papers ${ }^{89} 10$ and those to be presented here show, however, that the radiation emitted by a given tube supplied by a generator of any voltage wave form has a quality (full absorption curve) nearly identical with that which could be obtained with some constant potential generator operating at a particular voltage. Further it is found that, with different generators operating to give the same quality, the intensity is more nearly proportional to the effective tube current than to the average tube current.

An X-ray tube, on a given constant voltage, emits a radiation of definite spectral distribution which is independent of the tube current; as the current increases the radiation increases alike for all wave lengths. The ionization chamber measures the number of roentgens per unit time which, in this case, are proportional to the intensity. As the applied voltage is increased, a larger fraction of the intensity of the X-ray beam falls in the higher frequency (shorter wave length) intervals. The intensity has increased more than the number of roentgens per unit time as measured by the ionization chamber; and the number of roentgens measured has increased more than the

1 Deviation from this proportionality occurs only for X-ray beams of such great intensity that columnar ionization takes place. This is seldom encountered in practice.

2 L. S. Taylor, B.S.Jour. Research, vol. 5 (RP212), p. 517, 1930.

3 This is commonly given by plotting the logarithm of the percent transmitted, as indicated by the ionization chamber, against the thickness of the absorbing medium.

E. A. Pohle and C. S. Wright, Radiology, vol. 14, p. 17, 1930.

$\checkmark$ L. S. Taylor, Radiology, vol. 16, p. 302, 1931.

- R. B. Wilsey, Radiology, vol. 17, p. 700, 1931

7 L. Silberstein, Phil. Mag., ser. 7, vol. 15, p. 375, 1933.

8 L. S. Taylor and K. L. Tucker, B.S.Jour. Research, vol. 9 (RP475), p. 333, 1932.

- L. S. Taylor, G. Singer, and C. F. Stoneburner, B.S.Jour. Research, vol. 9 (RP491), p. 561, 1932.

10 L. 8. Taylor, Strahlentherapie (in press). 
dosage value of the radiation. Similarly, when a pulsating voltage wave form is used to excite the tube, the quality of the radiation fluctuates during the cycle from the softer initial value to the harder crest value during the wave period, and adds up to some average spectral distribution which the operator seeks to characterize in some definite and simple way. Experiment shows this composite spectral distribution for the common types of X-ray generator to be equivalent to that produced by the particular constant potential, which produces a radiation having also the same absorption curve. Comparing mechanical rectifiers (full wave) with a constant potential excitation, $150 \mathrm{kv}$ constant potential caused a radiation having the same absorption curve as produced by $190 \mathrm{kv}$ (peak) and by $200 \mathrm{kv}$ (peak) respectively, on two mechanical rectifiers; and this similarity extended over widely different values of the peak voltages. It was also found that these two mechanical rectifiers were, in fact, operating at $150 \mathrm{kv}$ effective (rms). ${ }^{11} \quad$ Furthermore, for any other given value of the effective voltage the absorption curves for all three generators were found closely alike for filtrations up to $1.2 \mathrm{~mm}$ of copper.

In addition, for the same effective voltage, the intensity of the radiation, as measured by air ionization, per effective milliampere of tube current, was practically the same for the different types of generator, even when the load distorted the voltage wave form. Having established by the resultant absorption curve that the quality of the radiation from different types of generator is the same when the effective voltage is the same, it is sufficient, for comparing heterogeneous X-ray beams or X-ray generators, to measure the radiation intensity at one filtration only and at different effective voltages. That is, two generators yielding the same output per milliampere, at a given filtration and given effective voltage, will function closely alike at other effective voltages and filtrations. This was found to hold with the types of generators used, for filtrations ranging from 0.1 to $1.2 \mathrm{~mm}$ copper. It should be added that, for the same effective voltage, the output per effective milliampere of the tube is alike not only as regards air ionization and absorption curve, but also was found alike as regards percentage depth intensity in media of low atomic number.

The need felt for specifying the quality of the radiation by a single magnitude is thus satisfied by stating the constant voltage which will produce it; and, in addition, the in tensity of the radiation having that quality is given as more nearly proportional to the effective than to the average tube current. In fact, it was found that the output per average milliampere varied with peak voltage to such an extent as to be without real significance in most cases. This fact is undoubtedly responsible in a large measure for the very great discrepancies between comparisons of X-ray tubes and generators by various observers, many of whom have been prone to compare generators or tubes under widely different load conditions, then correcting the various outputs on the assumption that there is a linear relationship between X-ray output and average tube current. For the usual X-ray transformer having characteristically large electrical regulation, this assumption is far from correct.

Another common error has been introduced by improper control of such factors as the capacitance of the aerial system, diaphragming of the X-ray beam, and the type of tube enclosure. Tests made in con-

11 See footnote 9 , p. 294. 
nection with this and past studies ${ }^{12}$ have shown that a variation of any one of these factors influences the final result and it is thus essential that all such factors remain constant throughout any adequate comparison of X-ray tubes or generators.

As a continuation of the study outlined in the foregoing paragraphs, the main objects of the present paper are:

1. To compare the radiation from a single X-ray tube operated on several types of voltage wave form;

2. To compare the effects of different criteria of control of applied voltage and tube current;

3. To show how various radiations may be equated to constant potential radiation.

\section{EXPERIMENTAL PROCEDURE}

The following types of high voltage X-ray generator were employed: A double disk mechanical rectifier, $\mathrm{B},{ }^{13}$ having a divided high tension transformer (two transformers in a single tank) and rectifying over approximately $20^{\circ}$ of the half cycle; a commercial "constant potential" generator, $\mathrm{C}$, having a ripplage ${ }^{14}$ of about 2 percent per milliampere; a "constant potential " generator, F, (B.S. standard) having a ripplage of about 0.2 percent per milliampere; a half-wave kenotron rectifier, $\mathrm{D}$, using the same two-pole high tension transformer previously used for generator, $\mathrm{A}$; a full-wave kenotron rectifier, $\mathrm{E}$; and a half-wave kenotron rectifier, G; using the same high tension transformer as generator, E.

A single type of thin walled cerium glass Coolidge tube was used throughout this study.

All generators were so arranged that they could be connected to the same aerial system leading to the X-ray tube (fig. 1). An interchange of generators could be accomplished in a minute's time so as to eliminate any short-time change that might occur in the tube. Numerous check runs made at intervals over several months showed no change in the X-ray tube used. An air blast from a high pressure blower was directed against the tube walls for cooling. Half-megohm resistors were used in series at all times in each side of the high-tension line for suppressing any surges or high-frequency oscillations.

Average and effective (rms) tube currents were measured in the manner previously described.15 For the effective current measurements a thermo-millammeter $\mathrm{B}$ was used. To protect the heating element from damage due to surges it was connected into the circuit through $1_{32}^{\prime}$ ampere Littel fuses. In addition, the meter and fuses were short-circuited when not actually being read.

12 See footnote 8, p. 294.

13 The letter designation of generators $\mathrm{A}, \mathrm{B}$, and $\mathrm{C}$ correspond to those used in earlier publications involving the same generators under the same condition of aerial, tube enclosure, etc. Hence the results given here are for the most part directly comparable with those given in the papers cited earlier.

14 Up to the present the term "constant potential" has been used in describing the potential supplied by kenotron or other valve tube rectification in which there is, of course, a slight ripple. A more accurate designation of voltages not actually constant but fluctuating about some mean value is "ripple voltage." Thus by a "ripple quantity" (potential or current) is meant a periodic quantity

$$
y=V_{0}+V_{1} \sin \left(\omega x+\alpha_{1}\right)+V_{2} \sin \left(2 \omega x+\alpha_{2}\right)+--
$$

in which the constant term $V_{0}$ is so large that all values of the quantity are positive (or negative). The amount of ripple ("ripplage" or "ripplance") in a ripple quantity is the ratio to the average value of the difference between the maximum and minimum values of the quantity.

10 See footnote 9 , p. 294. 
Average and effective (rms) tube voltages were measured, respectively, with D.C. and A.C. voltmeters used in conjunction with a 150 -megohm noninductive shielded resistor $\mathrm{R}^{16}$ placed across the hightension leads to the tube. The meters were connected in the center of the resistance so as to be near ground potential. This voltage measuring unit never drew more than $1 \mathrm{ma}$ from the circuit and hence had no undesirable effect on the operation of the generators.

The tube output was measured in roentgens per minute by means of a calibrated thimble ionization chamber kept in a fixed position with respect to the filters and tube. The distance from the center of

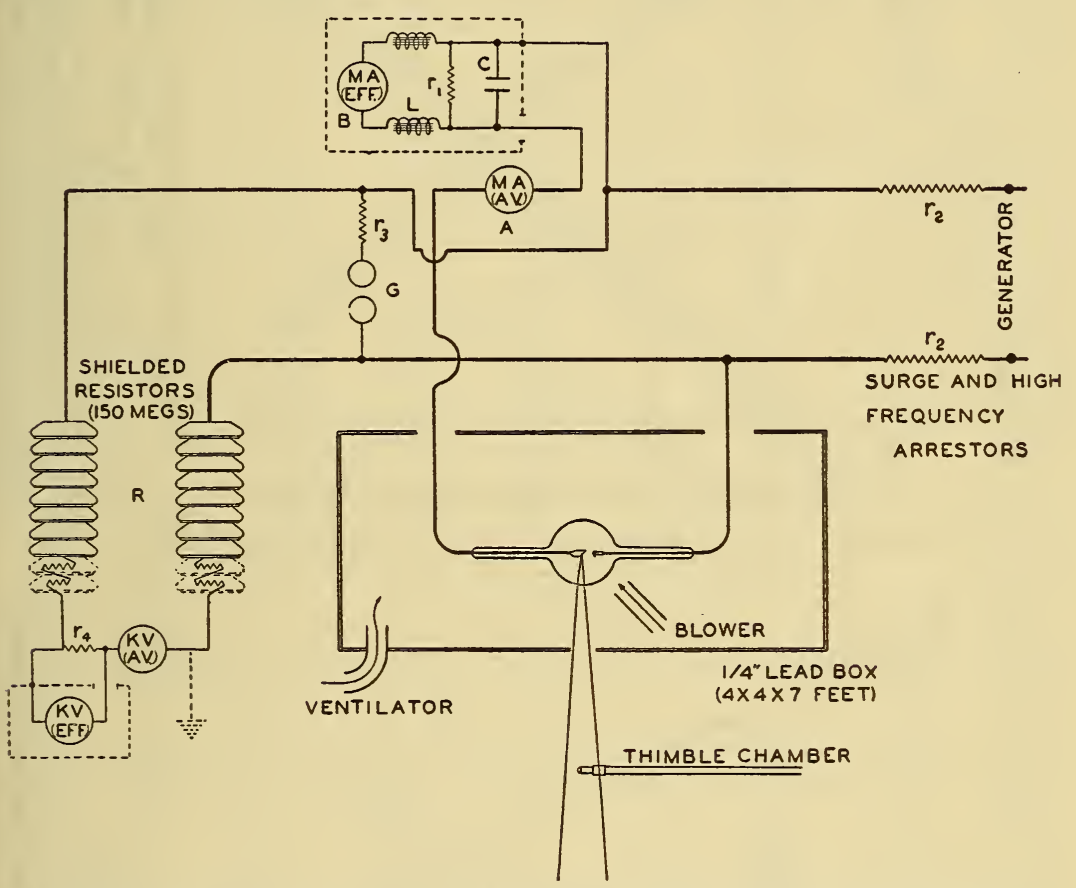

FIGURE 1.-Diagram of apparatus showing voltage and current measuring equipment.

the chamber to the center of the focal spot was $50 \mathrm{~cm}$, and at $15 \mathrm{~cm}$ from the chamber were placed the limiting aperture and a permanent filter (except during the determination of absorption curves) of $0.557 \mathrm{~mm}$ of copper, plus $1 \mathrm{~mm}$ of aluminum ${ }^{17}$ on the side next to the chamber. Earlier tests showed that secondary radiation from the filter at this distance had no influence on the ionization measurements. The diameter of the beam at the chamber was $5.5 \mathrm{~cm}$ throughout. To show the effect of the cross sectional area of the beam upon the measurements, it was found that when a beam diameter of $14.5 \mathrm{~cm}$ was used there was an increase in ionization of $9.0 \%$ for all voltages applied to the tube. This increase may be ascribed to the inclusion of stem radiation which had been cut off by the smaller aperture. In order that measurements made at different

1 L. S. Taylor, B.S.Jour. Research, vol. 5 (RP217), p. 609, 1930.

17 Compare with $0.535 \mathrm{~mm}$ Cu filtration used in our earlier papers. 
times might be directly comparable. all ionization current readings were corrected to a normal atmospheric condition of $22^{\circ} \mathrm{C}$. and 76 $\mathrm{cm}$ mercury pressure.
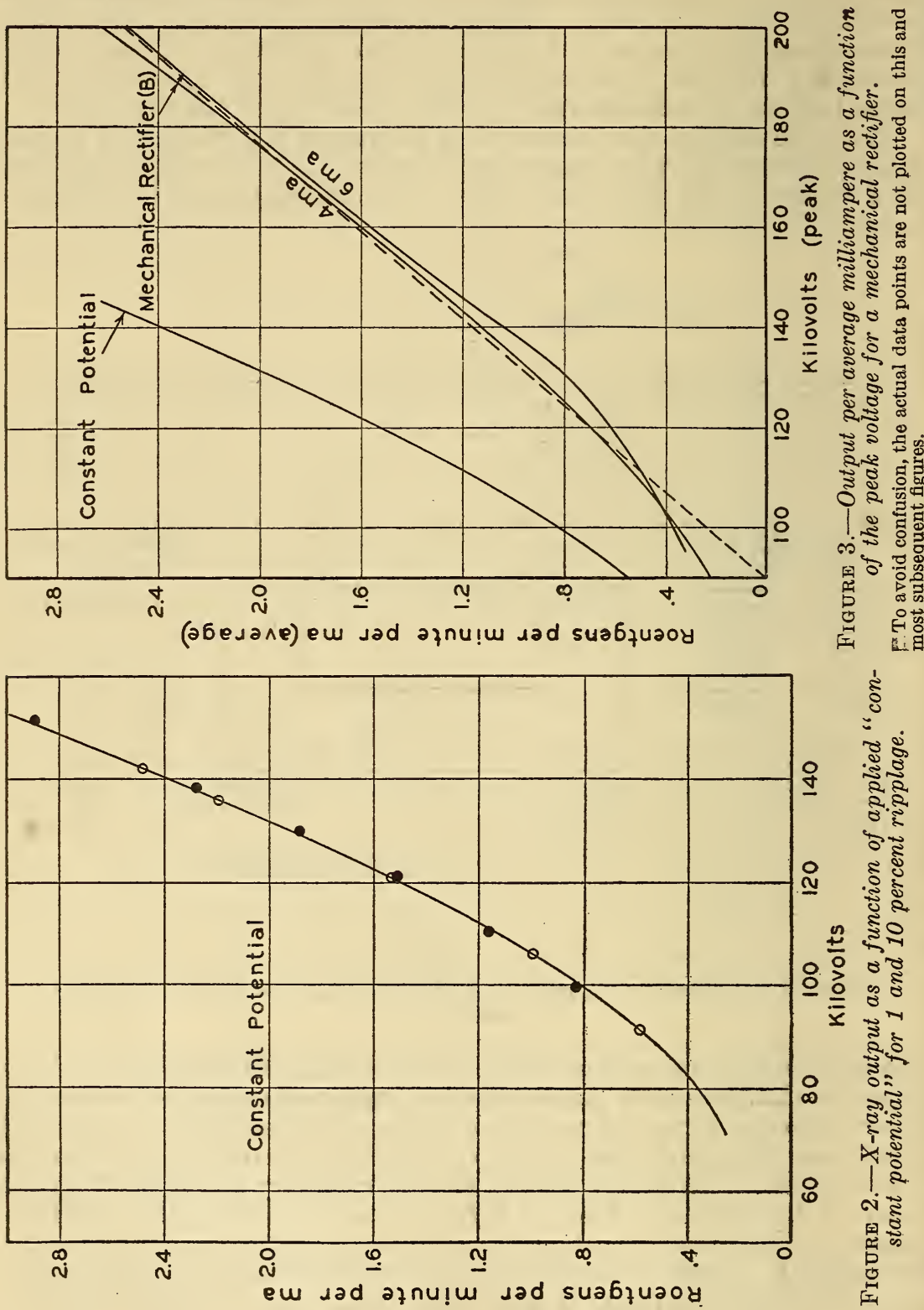

III. EXPERIMENTAL RESULTS

1. OUTPUT PER AVERAGE MILLIAMPERE

Outputs per unit tube current were usually determined from two operating currents, 4 and $6 \mathrm{ma}$, to bring out the differences produced by changes in the tube load. Similar measurements made for smaller 
and larger tube currents show that the order of magnitude of the change in output per average milliampere with tube current is about the same at all currents up to $10 \mathrm{ma}$.

Figure 2 shows, for the two constant potential generators $\mathrm{C}$ (dots) and $\mathrm{F}$ (circles), the output per average milliampere as a function of
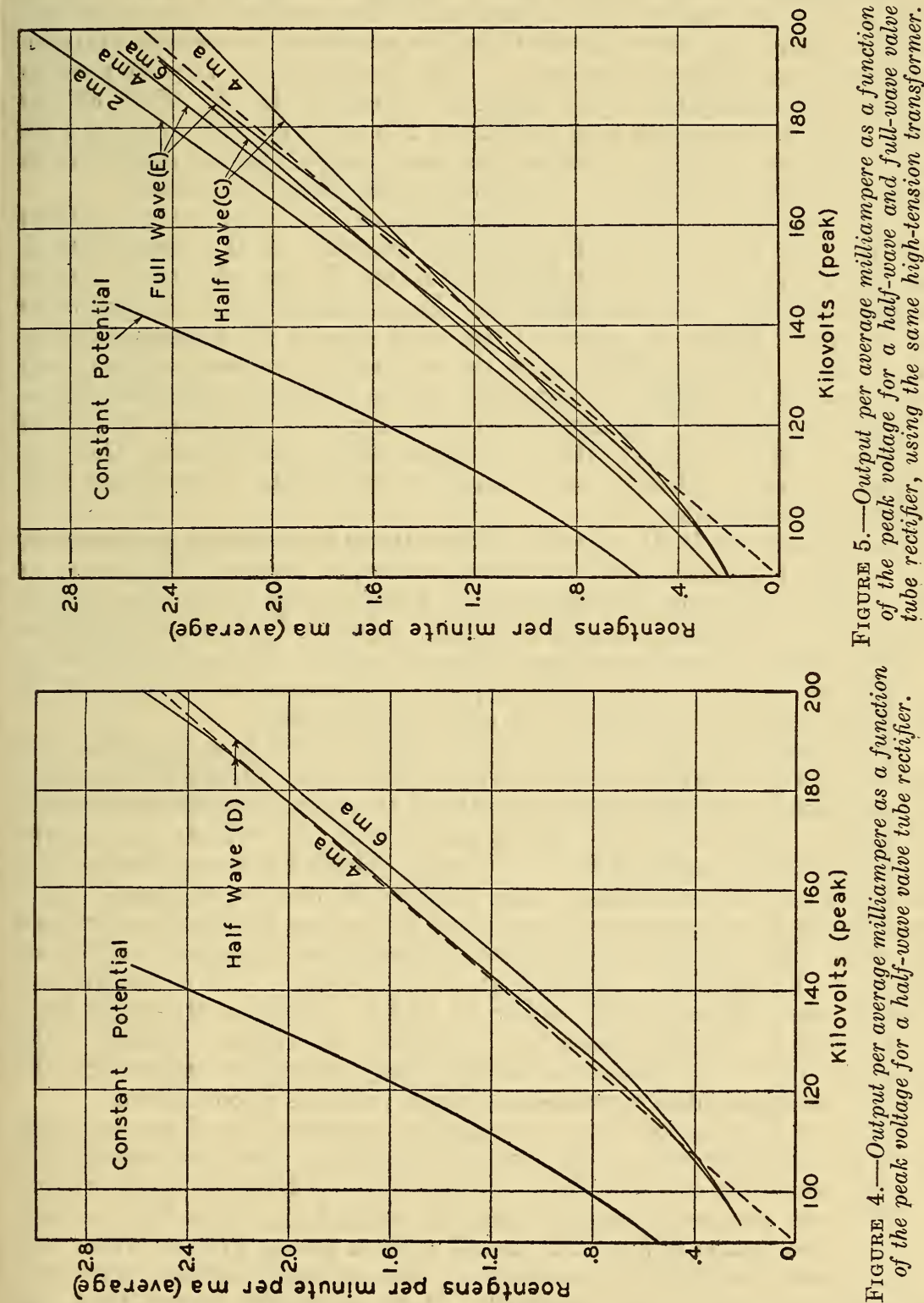

the peak voltage. The ripplage of these generators was about 10 and 1 percent, respectively, at the tube currents used. It will be noted, however, that the points for the two generators fall very closely along the same curve. This bears out previous findings that, 
for the type of measurement encountered in therapeutic X-ray work, the difference in X-ray output between 1 and 10 percent ripplage in constant potential generators is not greater than 1 or 2 percent and is consequently of no significance.

The average and effective tube currents in figure 2 were almost identical, as, of course, they should be. The peak voltages measured with a sphere gap were higher than the effective or average voltages by about the expected amount. This accounts for the 10 percent ripplage in generator $C$ as against 1 percent in $F$. For closest agreement between the two generators $F$ and $C$, effective rather than peak voltages should, of course, be used, although the difference is seen to be small enough to be neglected for many purposes.

The output per average milliampere for the mechanical rectifier $B$ is given by the curves in figure 3 as a function of the peak voltage. Similar curves for the half-wage generator $D$ are given by curves in figure 4 ; and for the full-wave, $E$, and half-wave, $G$, generator by curves in figure 5 . Curve $C$ on each plot is for constant potential. The straight dashed line is the same on all curves and is purely for reference to assist in comparing curves between different groups.

The first fact of significance is the same as reported in similar earlier studies, ${ }^{17}$ namely, the very great difference between the output per average milliampere for constant potential as compared with all other wave forms.

For generators $B, D, E$, and $G$ the outputs per average milliampere are grouped within a comparatively narrow region. However, as the voltage increases the divergences between the several generators seem to be increasing, as well as the divergences between the outputs of the same generator under different loads. In fact, at $200 \mathrm{kV}$ (peak) there is, based upon this type of comparison, an output spread of about 25 percent between the several generators.

The variations between generators are in part due to differences in electrical regulation of the main transformer. Hence, to compare the half-wave and full-wave generators, the same transformer should be used. This was done with generator $E$, for which the output curves are given in figure 5 . The results, which have been thoroughly checked, are not consistent with respect to load conditions. At 4 ma (average) tube current there is an increasing divergence between the half- and full-wave generators, beginning at 1 percent at $130 \mathrm{kv}$ (peak) and reaching 17 percent at $190 \mathrm{kv}$ (peak). At 6 ma there is a decreasing divergence in going to higher voltages, changing from 17 percent at $130 \mathrm{kv}$ (peak) to 1 percent at $190 \mathrm{kv}$ (peak). The impossibility of accurately comparing X-ray generator outputs on the basis of average tube currents and peak voltages is confirmed.

There is thus no obvious relationship between the X-ray outputs even when operated on the same transformer and accordingly in comparisons it is not strictly necessary to use identical transformers.

The only generalization that can be made from these data is that, if the tube currents are not varied over a range greater than \pm 2 milliampere (average), the outputs of the various generator types will be the same within \pm 15 percent. It is moreover found that the output per average milliampere depends upon the particular tube current to such a degree as to render meaningless all comparisons

\footnotetext{
17 See footnote 8, p. 294.
} 
between tubes and generators unless carried out at the same average current.

Many observers have been prone to compare generators or tubes under widely different load conditions, correcting the various outputs

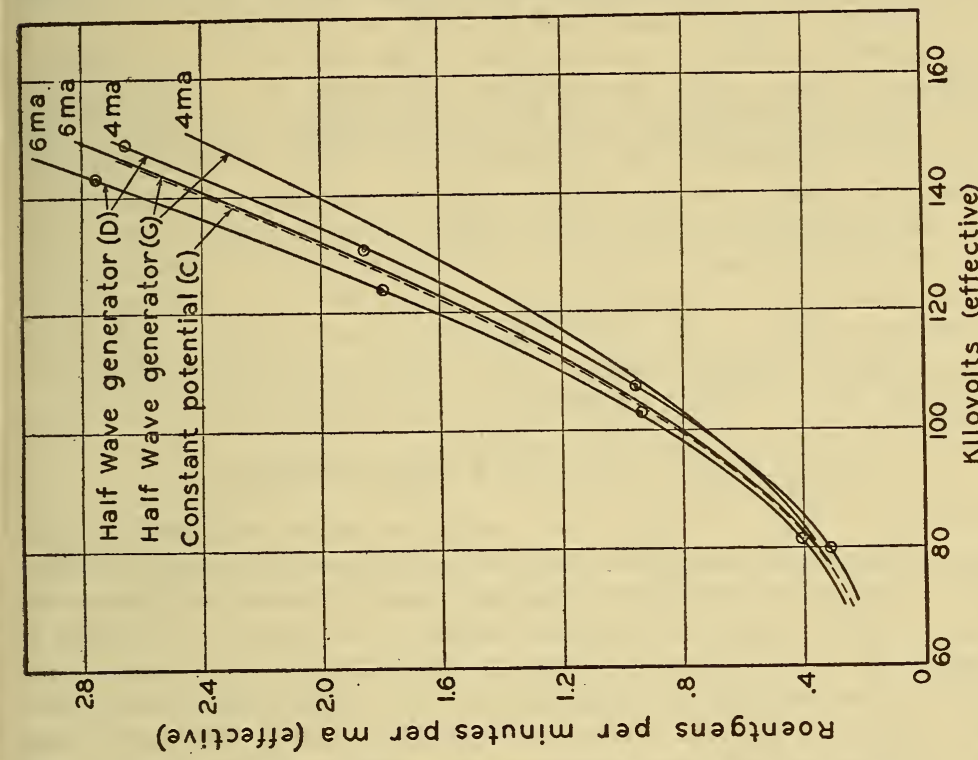

ริ ॐ

西

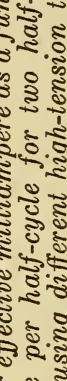

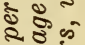

३)

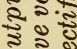

2.

$\sqrt[3]{0} \frac{\pi}{2}$

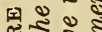

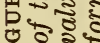

至

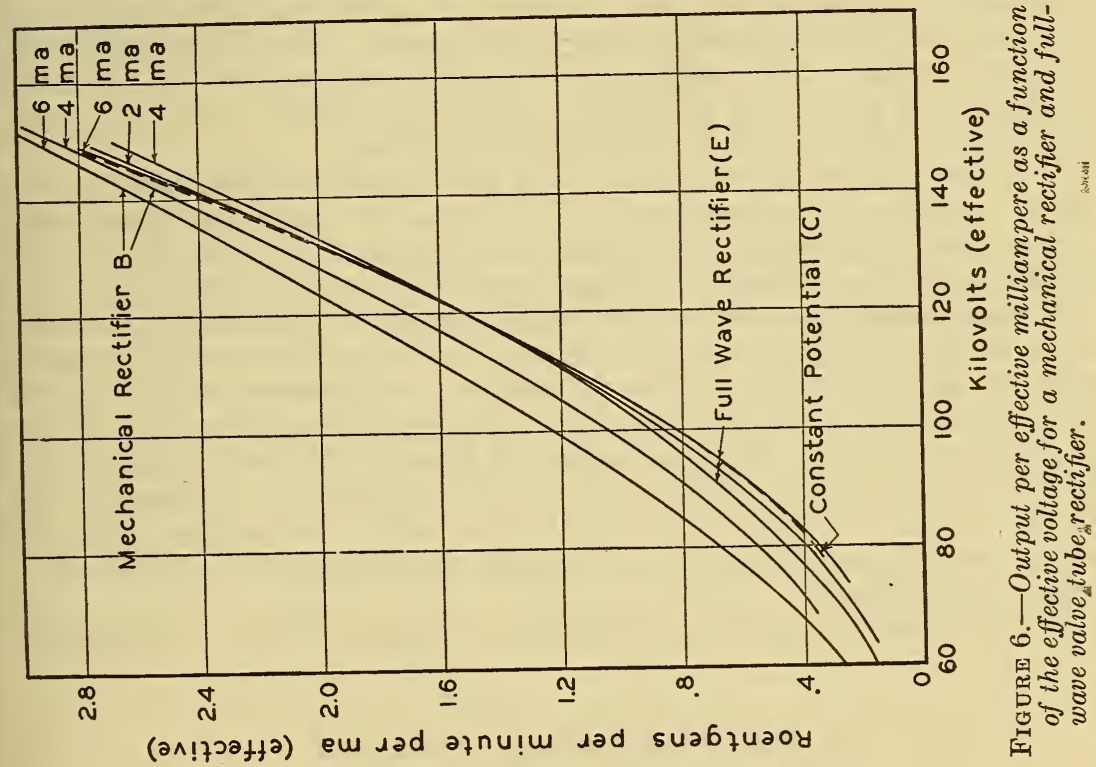

on the assumption that there is a linear relationship between $\mathrm{X}$-ray output and average tube current. For the usual X-ray transformer, having characteristically poor electrical regulation, this assumption is far from correct. For example, it is not permissible to assume that, if the outputs of two generators at a given peak voltage differ by 20 
percent, the difference can be made up by increasing the tube current of one by 20 percent, keeping the same peak voltage.

The observed difference in output per average milliampere between the half- and full-wave generator should ideally be nil, since to obtain the same average tube current with a half-wave generator as for a full-wave generator, over a number of cycles, the current during a single half-cycle need only be doubled throughout. However, in practice, a marked difference is observed principally because of the large electrical regulation of the transformers. For, with the necessarily larger instantaneous values of the tube current in the half-wave generator, the resultant voltage wave form becomes so distorted as to produce a marked influence upon the X-ray output. Furthermore, some X-ray tubes are purposely constructed, by such a disposition of the focussing shield, as to limit the space charge effect, and so to pass only a narrow current wave. With such a tube the difference between a full- and half-wave generator is more marked.

\section{OUTPUT PER EFFECTIVE MILLIAMPERE}

Effective (rms) tube currents and voltages were obtained at the same time as the data just presented, hence, are entirely comparable therewith. Values of the X-ray output per effective milliampere, as a function of the applied effective voltage, are plotted in figure 6 for generators $\mathrm{B}, \mathrm{C}$, and $\mathrm{E}$. The broken line curve, $C$, is for constant potential. It is seen that, as with the earlier reports, the curves for the full-wave generator $\mathrm{E}$ follow very closely along the curve for constant potential while those for the mechanical rectifier $B$ approach the constant potential curve at higher voltages. At $150 \mathrm{kv}$ (effective) the total spread of the output per effective milliampere for all three generators is less than 10 percent; while, for either B or E alone, with constant potential, it is less than 4 percent.

It is thus indicated that the output per effective milliampere at the higher voltages is approximately the same for full-wave generators and constant potential. The previous studies show that the X-ray quality for given effective voltages would likewise be the same.

Measurements of the effective (rms) voltage in half-wave generators have little significance except under ideal conditions which seldom exist in X-ray circuits. This is because the effective voltage meter gives a root-mean-square value over a whole cycle while only half the cycle is effective in the production of X-rays - the other half being suppressed by the rectifier. If it were possible to measure the effective (rms) voltage during only the useful half-cycle, then the results would undoubtedly be directly comparable with those for a full-wave rectifier.

For a full-wave rectifier the effective voltage $E$ (r.m.s.) is given by

$$
\begin{aligned}
\left.E_{2 / 2} \text { (r.m.s. }\right) & =E(\text { peak }) \sqrt{\frac{\int_{0}^{2 \pi} \sin ^{2} \omega t d t}{2 \pi}} \\
= & \frac{\sqrt{2}}{2} E(\text { peak })=0.71 E(\text { peak })
\end{aligned}
$$


For a pure sine wave this may be divided into two parts

$$
\left.E_{2 / 2} \text { (r.m.s. }\right)=E(\text { peak }) \sqrt{\frac{\int_{0}^{\pi} \sin ^{2} \omega t d t+\int_{\pi}^{2 \pi} \sin ^{2} \omega t d t}{2 \pi}}
$$

If, however, we consider one half of the wave to be suppressed, and therefore useless, we have for a half-wave sine generator

$$
\begin{aligned}
\left.E_{1 / 2} \text { (r.m.s. }\right) & =\frac{\sqrt{2}}{2} E_{2 / 2} \text { (r.m.s.) }=0.71 E_{2 / 2} \text { (r.m.s.) } \\
& =0.5 E \text { (peak) }
\end{aligned}
$$

We may apply this result to the ideal case of a half-sine wave and full-sine wave voltage of the same peak value. It would be observed that the rms voltage read over the full cycle on the half-wave generator if multiplied by $\sqrt{2}$ will be the same as the r.m.s. voltage read on the full-wave generator.

The voltage wave form, however, in the usual X-ray generator is not a pure sine wave (sec. 4) because of the poor electrical regulation of the transformer and the reaction of the $\mathrm{X}$-ray tube. Analysis of the actual wave form by Fourier series would show each useful half wave to correspond to a wave form having a predominant third harmonic and less important harmonics of higher order. We have then a complex wave form for which the r.m.s. voltage is no longer given by equation (2) but rather by the following:

$$
E_{27_{2}} \text { (r.m.s.) }=\frac{1}{\sqrt[3]{2 \pi}} \sqrt{\int_{0}^{\pi}\left[\Sigma E_{b} \sin \left(b \omega t+\theta_{b}\right)\right] d t+\int_{\pi}^{2}\left[\Sigma E_{b} \sin \left(b \omega t+\theta_{b}\right)\right] d t}
$$

Solution of this equation for one half wave only, shows that equation (3) relating the r.m.s. value during a half cycle to the r.m.s. value over a full cycle is valid only when there are no strong even harmonics present. Since, as pointed out, the wave form is composed predominantly of the first and third harmonics, we can, to a first approximation, obtain a measure of the effective voltage during the useful part of the cycle by multiplying the measured value by $\sqrt{2}$. This type of correction is of course unnecessary for any phenomena utilizing the full cycle of the voltage wave, which includes all other common $\mathrm{X}$-ray generators.

By a similar procedure, we should also correct the effective (r.m.s.) tube current readings to apply only during the useful half of the cycle. Making the same assumptions for the current wave form as were made for the voltage wave form, it is found that the r.m.s. current values over a full cycle as read on the meter should be reduced by a factor of $\sqrt{1 / 2}$ which is equivalent to increasing the output per effective milliampere during the useful half cycle by the factor $\sqrt{2}$.

Outputs per effective milliampere, during the useful part of the cycle, obtained for the two half-wave generators D and G, are plotted in figure 7 after the measured tube currents, and voltages were thus corrected by the factors $\sqrt{\gamma_{1}}$ and $\sqrt{2}$, respectively. The broken line curve is for the same tube on constant potential. While the agree- 
ment between the three generators is not as close as in the case of the full-wave generators, the spread of the outputs, at a given voltage, is not nearly so large as in the case of equal peak voltages (figs. 4 and 5). As shown below, the quality of these radiations at any given effective voltage is very nearly the same, thus bearing out our earlier findings on other generators.

\section{QUALITY AS RELATED TO VOLTAGE MEASUREMENTS}

Ordinarily it has been assumed that at a given peak voltage, copper absorption curves obtained under different tube currents will be the same. This is based upon the further assumption that the output per average milliampere of a tube does not vary with the load. We have just shown, in the case of all but constant potential gener-

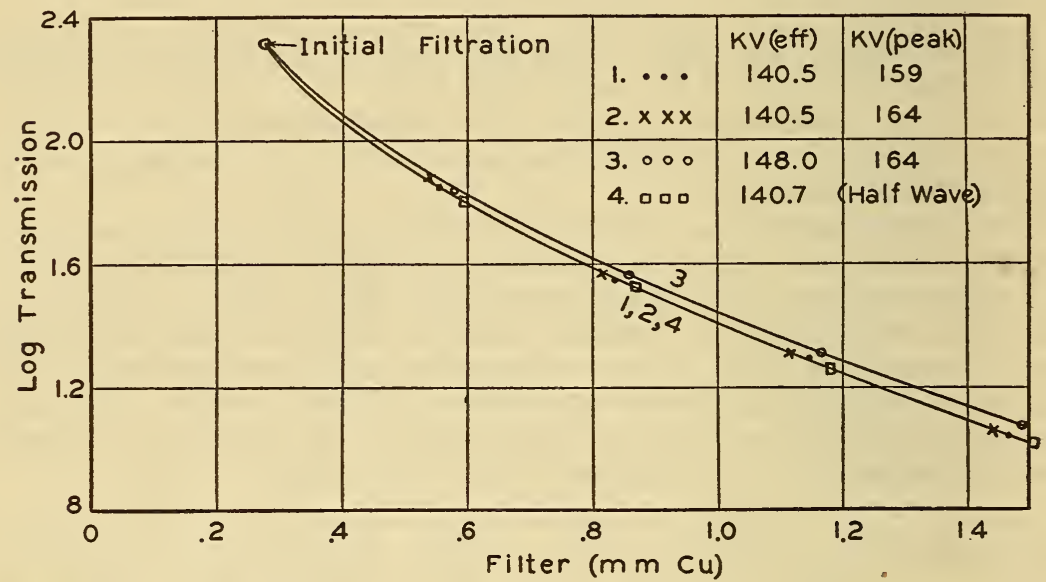

FIGURE 8.-Copper absorption curves showing the relationship between the $X$-ray quality and the effective voltage as the tube load is changed.

ators, that this assumption does not hold for changes in tube current even as small as 2 ma. On the other hand, it has been shown that at any tube current the quality of the $\mathrm{X}$-ray beam is the same at a given effective (rms) voltage. ${ }^{18}$

The curves in figure 8 show a very sensitive test of the relationship of quality to voltage. These were made with the full-wave generator $\mathrm{E}$, since in this case variations in tube current had the least effect on the output per milliampere. Curves 1 (dots) and 2 (crosses) were obtained, respectively, with the same effective voltage (140.5) but with peak voltages of 159 and $164 \mathrm{kv}$ the peak voltage change being caused by a change in tube current of 2 to 4 ma, respectively. Curves 2 and 3 (circles) were for the same peak voltage $(164 \mathrm{kv})$ but with respective effective voltages of 140.5 and $148 \mathrm{kv}$, and respective tube currents of 4 and 2 ma. From these we see that $(a)$ so long as the effective voltage is maintained constant, the absorption curves (hence X-ray quality) are the same (curves 1 and 2); (b) a change in tube current of $2 \mathrm{ma}$, with the same peak voltage, causes an appreciable change of quality (curves 2 and 3 ).

\footnotetext{
18 See footnote 9, p. 294.
} 
Similar results were obtained using the half-wave generator $\mathrm{D}$, after making correction as in section (b) for the suppressed half of the cycle which is ineffective in X-ray production. Curve 4 (squares) was obtained with the generator (D) operated at $99.7 \mathrm{kv}$ (effective, measured) or $140.5 \mathrm{kv}$ (effective, corrected) showing a very close agreement between the qualities obtained with it and the full-wave generator $(\mathrm{E})$.

Finally, figure 9 gives the copper absorption curves for generators $\mathrm{F}, \mathrm{B}$, and $\mathrm{D}$ at approximately the same effective voltage but at widely different peak voltages, and shows the close relationship between the effective voltage and quality. The operating conditions

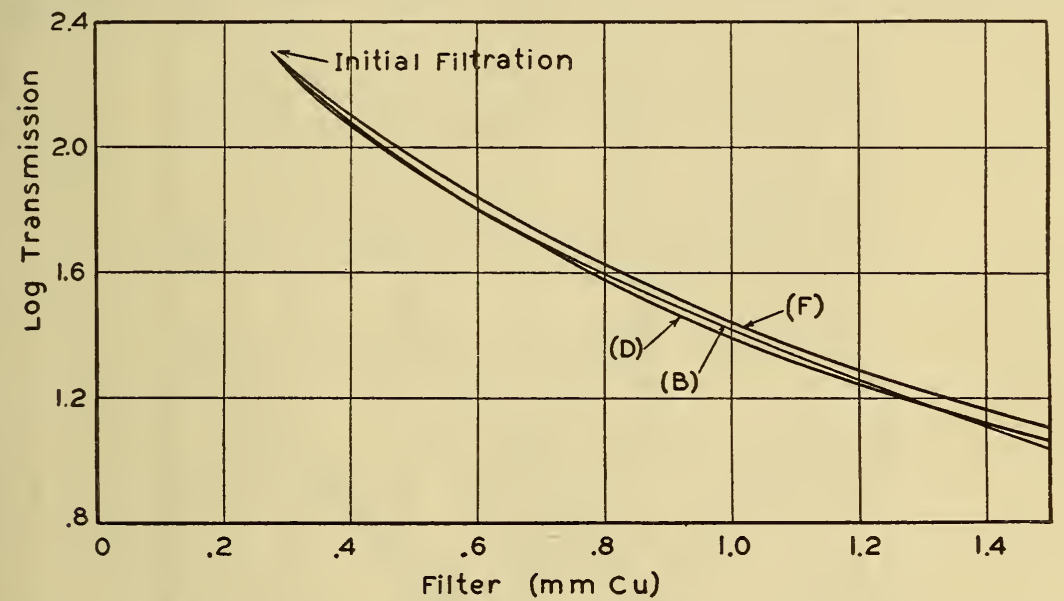

FIGURE 9.-Copper absorption curves for three generators of different type, operated at approximately the same effective voltage.

Generator F, $151.7 \mathrm{kv}$ (effective), $153 \mathrm{kv}$ (peak).

Generator B, $153 \mathrm{kV}$ (effective), $192 \mathrm{kV}$ (peak).

Generator D, $154 \mathrm{kV}$ (effective), $180 \mathrm{kV}$ (peak).

are given in the legend. These curves show the qualities of the radiations to be the same; in section $(b)$ it was found that the outputs per effective milliampere were practically the same, so it may be concluded that the radiations are essentially alike.

\section{CHANGES IN VOLTAGE WAVE FORM}

Changes in the voltage wave form, brought about largely by poor electrical regulation of the transformer, appear to be the principal cause of the irregularity reported above. Information regarding the wave shape of the voltage applied to the tube may be obtained from the measurements already recorded, so that oscillographic examination of the wave form, which is laborious under such varied conditions, need not be resorted to.

The general wave form may be inferred from the relationship between the effective (rms) and peak voltage. These are given in figure 10 for the half-wave generator (D) and in figure 11 for the halfwave $(G)$ and full-wave $(E)$ generator employing the same transformer. ${ }^{19}$ The heavy straight line on each plot is for the case of a

10 Similar curves for generator B are given in figure $7(B)$ of B.S. Research Paper No. 491.

$205-33-10$ 
pure sine wave where $\mathrm{kv}$ (peak) $=\sqrt{2} \mathrm{kv}$ (effective). It is evident that, where the curve for a particular generator and tube current lies below the line for a sine wave, a relatively broad flat-topped voltage wave form is indicated which approaches constant potential as the limit. Where the curve lies above the line $\mathrm{kv}$ (peak) $=\sqrt{2} \mathrm{kv}$ (effective), a relatively narrow or peaked voltage wave form is indicated.

Thus we find that if the tube current at a given peak voltage is increased, there is a marked decrease in the effective voltage, indicating a narrowing of the voltage wave form and causing a corresponding change in quality (a change corresponding to a lower constant poten-

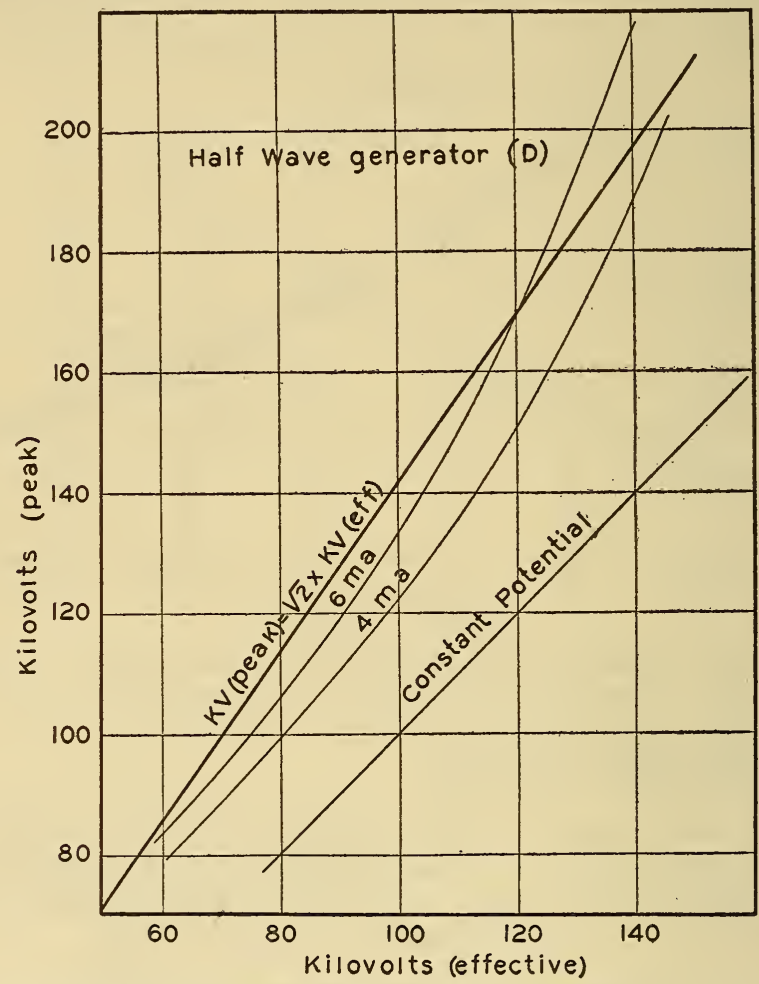

FIGURE 10.-Relationship between effective and peak voltage per half cycle for a half-wave rectifier

tial). The effect on a given transformer in passing from full-wave to half-wave rectification is indicated by the curves in figure 11. For a given average tube current of $4 \mathrm{ma}$ and the same peak voltage, an increase in the effective voltage in going from full- to half-wave is found. This indicates a broadening of the wave form and an increase in the hardness of the radiation; which is to be expected, since the half-wave generator draws a larger maximum tube current during the cycle and flattens out the wave.

These curves likewise show to what extent we may rely upon the assumption involved in equation (3) and used in arriving at the effective voltage in the case of half-wave generators. For example, it is found in figure 11 that the $6 \mathrm{ma}$ curve for generator $\mathrm{G}$ approaches nearer a sine wave value than the 4 ma curve; hence should yield 
more nearly the normal output per milliampere at that effective voltage. This is found to be the case for the same 6 ma curve in figure 7.

\section{CONCLUSION}

The experimental results presented in this paper corroborate in detail and further extend our earlier findings regarding the relationships between X-ray tube output and the applied voltage and current supplied by different types of high-voltage generator, in the following essentials:

1. For a given effective (rms) tube voltage, the $\mathrm{X}$-ray outputs per effective milliampere of tube current for all $\mathrm{X}$-ray generators are

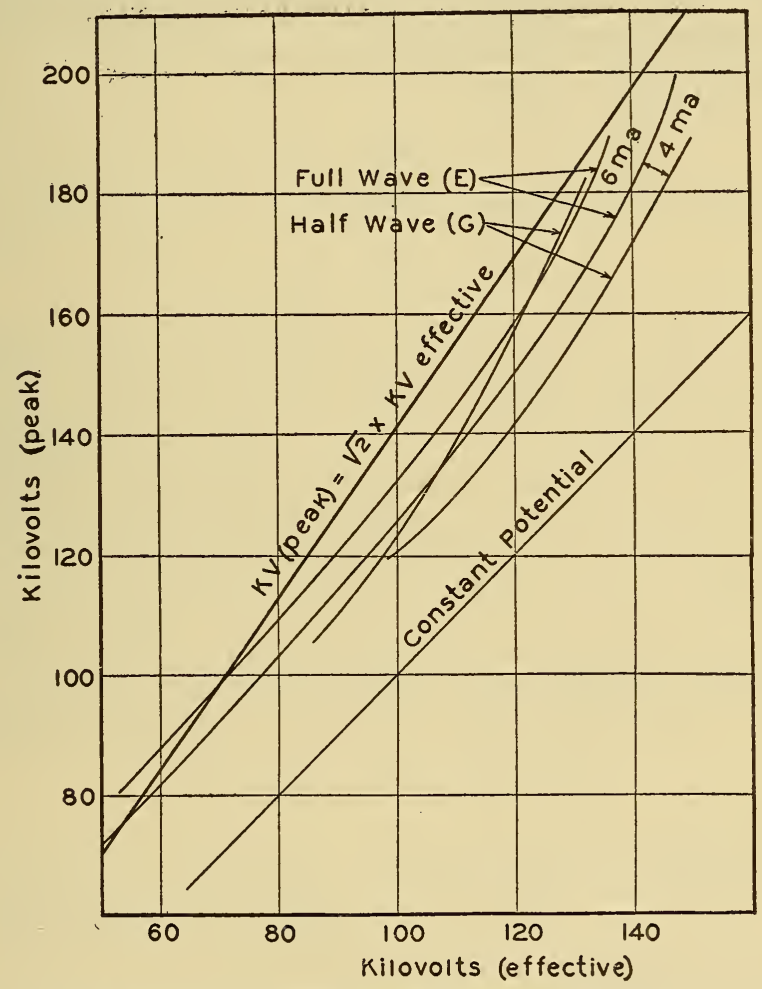

FIGURE 11.-Relationship between effective and peak voltage per half cycle for a half-wave and full-wave valve tube rectifier, using the same transformer.

much more nearly the same than for any other type of voltage and current measurement. The variation of output per effective milliampere with tube current is small; hence results obtained at one tube current are comparable with results obtained at some other tube current.

2. X-ray qualities at a given effective voltage are nearly the same for all generators. This means, in effect, that the output and quality of any generator may be reduced to terms of an equivalent output and quality obtained with constant potential which, in itself, is perfectly definite. 
3. Ripplage in constant potential generators of 1 to 10 percent causes no appreciable differences as far as concerns the usual quality and quantity measurements.

4. Effective (rms) current and voltage measurements on a halfwave generator must be suitably corrected for the useless half cycle when using the customary types of rms reading meters.

5 . In the comparison of half-wave and full-wave generators with constant potential at given peak voltages and tube currents, there is no simple relationship between the X-ray output or quality.

6 . The specification of X-ray output in terms of average tube current and peak voltage is indefinite because of voltage wave form distortion with change in load.

This investigation has been made possible through the cordial cooperation of the American X-ray equipment manufacturers, to whom we express our appreciation.

Washington, May 1, 1933. 